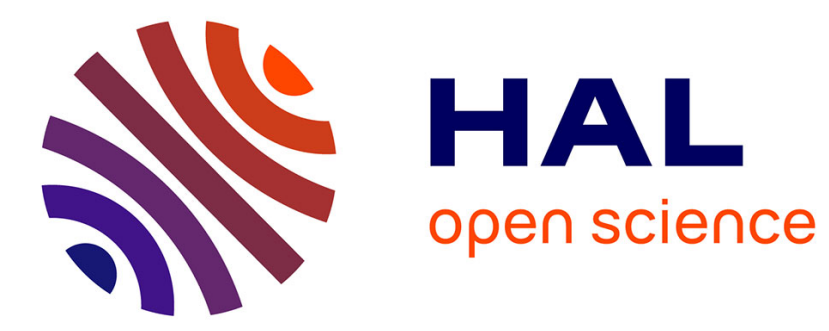

\title{
Periodic event-triggered control of nonlinear systems using overapproximation techniques
}

Dominicus Paulus Borgers, Romain Postoyan, Adolfo Anta, Paulo Tabuada, Dragan Nešić, Maurice Heemels

\section{- To cite this version:}

Dominicus Paulus Borgers, Romain Postoyan, Adolfo Anta, Paulo Tabuada, Dragan Nešić, et al.. Periodic event-triggered control of nonlinear systems using overapproximation techniques. Automatica, 2018, 94, pp.81-87. 10.1016/j.automatica.2018.04.019 . hal-01787486

\section{HAL Id: hal-01787486 \\ https://hal.science/hal-01787486}

Submitted on 7 May 2018

HAL is a multi-disciplinary open access archive for the deposit and dissemination of scientific research documents, whether they are published or not. The documents may come from teaching and research institutions in France or abroad, or from public or private research centers.
L'archive ouverte pluridisciplinaire HAL, est destinée au dépôt et à la diffusion de documents scientifiques de niveau recherche, publiés ou non, émanant des établissements d'enseignement et de recherche français ou étrangers, des laboratoires publics ou privés. 


\title{
Periodic event-triggered control of nonlinear systems using overapproximation techniques
}

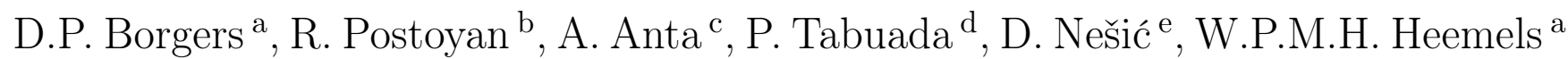 \\ ${ }^{a}$ Eindhoven University of Technology, P.O. Box 513, 5600 MB Eindhoven, The Netherlands \\ ${ }^{\mathrm{b}}$ Université de Lorraine, CRAN, UMR 7039, and CNRS, CRAN, UMR 7039, 2 avenue de la forêt de Haye 54516 \\ Vandoeuvre-lès-Nancy, France \\ ${ }^{\mathrm{c}}$ General Electric, Munich, Germany \\ ${ }^{\mathrm{d}}$ Cyber-Physical Systems Laboratory, Department of Electrical Engineering, University of California, Los Angeles, CA, USA \\ ${ }^{\mathrm{e}}$ Department of Electrical and Electronic Engineering, the University of Melbourne, Parkville, VIC 3010, Australia
}

\begin{abstract}
In event-triggered control, the control task consisting of sampling the plant's output and updating the control input is executed whenever a certain event function exceeds a given threshold. The event function typically needs to be monitored continuously, which is difficult to realize in digital implementations. This has led to the development of periodic event-triggered control (PETC), in which the event function is only evaluated periodically. In this paper, we consider general nonlinear continuous event-triggered control (CETC) systems, and present a method to transform the CETC system into a PETC system. In particular, we provide an explicit bound on the sampling period at which the event function is evaluated and we present a constructive procedure to redesign the triggering condition. The latter is obtained by upper-bounding the evolution of the event function of the CETC system between two successive sampling instants by a linear time-invariant system and then by using convex overapproximation techniques. Using this approach, we are able to preserve the control performance guarantees (e.g., asymptotic stability with a certain decay rate) of the original CETC system.
\end{abstract}

Key words: Event-triggered control; Digital implementation; Polytopic overapproximation; Nonlinear systems.

\section{Introduction}

In digital control applications, the control task consists of sampling the outputs of the plant and computing and implementing new actuator signals. This procedure is typically executed in a time-triggered fashion, which

\footnotetext{
^ This work is supported by the Innovational Research Incentives Scheme under the VICI grant "Wireless control systems: A new frontier in automation" (No. 11382) awarded by NWO (The Netherlands Organization for Scientific Research) and STW (Dutch Science Foundation), by the ANR under the grant COMPACS (ANR-13-BS03-0004-02), and by the Australian Research Council under the Discovery Projects Scheme.

Email addresses: d.p.borgers@tue.nl (D.P. Borgers), romain.postoyan@univ-lorraine.fr (R. Postoyan), aantam@gmail.com (A. Anta), tabuada@ee.ucla.edu (P. Tabuada), dnesic@unimelb.edu.au (D. Nešić), m.heemels@tue.nl (W.P.M.H. Heemels).
}

may lead to a waste of communication and energy resources, as the execution of the control task is done irrespective of whether there actually is a need for a control update or not. To mitigate the unnecessary waste of resources, various event-triggered control (ETC) strategies have been proposed in the recent literature, see, e.g., $[3,5,7,11,13,16,17,22,24,25]$. In ETC, the control task is executed after the occurrence of an event, generated by some well-designed triggering condition, rather than after a fixed period of time, as in conventional periodic sampled-data control. In this way, ETC is capable of significantly reducing the number of control task executions, while retaining a satisfactory closed-loop performance.

A main implementation issue of ETC (for which we will use the term continuous event-triggered control (CETC) from here on) is that the event function has to be monitored continuously, which is difficult to realize on digital platforms. A solution to this problem is periodic event- 
triggered control (PETC), in which the event function is only checked periodically at fixed equidistant time instances, thereby enabling (easier) implementation on a digital platform. Note that PETC differs from standard periodic sampled-data control, as in PETC the event times are only a (specific) subset of the sampling times and can be aperiodic. Of course, event-triggered control schemes for discrete-time systems (e.g., $[4,6,8,15,18,27])$ can also be interpreted as PETC schemes, but these do not take into account the inter-sample behavior. In the past few years, various PETC strategies have been proposed, see, e.g., [9-11,13,21]. However, to the best of the authors' knowledge, there are hardly any design methods for PETC for nonlinear continuous-time systems. Two exceptions are the works in $[23,26]$. In [23], the sample-and-hold implementation of general hybrid controllers for nonlinear systems is analyzed, which covers the PETC implementation of a nonlinear CETC system as a subcase. These results ensure that, under general conditions, if a compact set is uniformly globally asymptotically stable (UGAS) for the original CETC system, then this property is semiglobally and practically preserved for the emulated PETC system by taking the sampling period sufficiently small. In the recent work [26], an approach has been proposed for the design of PETC state-feedback controllers to stabilize nonlinear systems, which ensures uniform global asymptotic properties and provides an explicit bound on the sampling period.

In this paper, we present a method to transform a general nonlinear CETC system into a PETC system which preserves the control performance guarantees of the given CETC system. Our method consists of two steps. First, we upper bound the evolution of the event function of the given CETC system between two successive sampling instants by a linear time-invariant (LTI) system. Based on this LTI system, we can formulate a redesigned event function for the PETC implementation which would involve checking an infinite number of conditions at every sample time. To overcome this issue, we use convex techniques to overapproximate the evolution of the LTI system over a sampling period, and end up with a redesigned event function which is implementable in practice.

In contrast to [23], our method provides an explicit bound on the sampling period, it fully preserves the control performance guarantees of the given CETC system, and is not limited to stability of a compact set a priori. Compared to [26], we do not focus on stabilization and we can cope with a larger class of triggering conditions. Preliminary results have been presented in [21], in which we were only able to approximately preserve the control performance guarantees of the given CETC system. In addition, the new results presented here are based on less stringent conditions compared to [21] (see Remark 3 below for more details).

Nomenclature. Let $\mathbb{R}=(-\infty, \infty), \mathbb{R}_{\geqslant 0}=[0, \infty)$,
$\mathbb{N}=\{1,2, \ldots\}$ and $\mathbb{N}_{0}=\{0,1,2, \ldots\}$. Given $N \in \mathbb{N}$, we denote the set $\{1,2, \ldots, N\}$ by $\bar{N}$. For a vector $x \in \mathbb{R}^{n}$, we denote by $\|x\|:=\sqrt{x^{\top} x}$ its 2-norm, and for a ma$\operatorname{trix} A \in \mathbb{R}^{n \times m}$, we denote by $\|A\|:=\sqrt{\lambda_{\max }\left(A^{\top} A\right)}$ its induced 2-norm. For a signal $w: \mathbb{R}_{\geqslant 0} \rightarrow \mathbb{R}^{n}$, we denote the right limit at time $t \in \mathbb{R}_{\geqslant 0}$ by $w\left(t^{+}\right)=\lim _{s \downarrow t} w(s)$, when it exists. The solution $z$ of a time-invariant dynamical system at time $t \in \mathbb{R}_{\geqslant 0}$ starting with the initial condition $z(0)=z_{0}$ will be denoted by $z\left(t, z_{0}\right)$ or simply by $z(t)$ when the initial state is clear from the context. The notation $\lfloor x\rfloor$ stands for the largest integer smaller than or equal to $x \in \mathbb{R}$.

\section{Problem statement}

We consider a nonlinear plant of the form

$$
\dot{x}(t)=f(x(t), u(t)),
$$

where $x(t) \in \mathbb{R}^{n_{x}}$ is the state and $u(t) \in \mathbb{R}^{n_{u}}$ is the control input at time $t \in \mathbb{R}_{\geqslant 0}$. We assume that we have designed a continuous event-triggered state-feedback controller for plant (1), given by

$$
\begin{aligned}
\hat{x}(t) & =x\left(t_{k}\right), \quad \text { for } t \in\left(t_{k}, t_{k+1}\right] \\
u(t) & =k(\hat{x}(t)) \\
t_{0} & =0 \\
t_{k+1} & =\inf \left\{t>t_{k} \mid \Gamma(x(t), \hat{x}(t), \chi(t))>0\right\},
\end{aligned}
$$

where the function $k(\hat{x})$ defines the feedback law, $\hat{x}$ is the state information available to the controller, and $\chi \in \mathbb{R}^{n_{\chi}}$ is used to capture other relevant variables such as timers, counters, or possibly even the state of an auxiliary dynamical system $[5,7,22]$. The event function $\Gamma$ is designed such that some desired control performance (e.g., asymptotic stability with a certain decay rate) is achieved as long as it remains non-positive along the system's trajectories.

Writing the triggering law as in (2c) allows us to consider various event-triggers considered previously in the literature, which we illustrate by the following two examples. In [24], the condition $\Gamma(x, \hat{x})=\gamma(\|\hat{x}-x\|)-\sigma \alpha(\|x\|) \leqslant 0$ (for specific functions $\gamma, \alpha$ and $\sigma \in(0,1))$ ensures that a Lyapunov function $V$ has a guaranteed decay rate $(1-\sigma) \alpha(\|x\|)$ along the solutions to system (2) (which guarantees global asymptotic stability of the system). In [5], we have that $\chi=(\tau, \kappa, \eta)$ (where $\tau$ is a timer, $\kappa$ a counter, and $\eta$ the state of an auxiliary dynamical system), and that the condition $\Gamma(x, \hat{x}, \chi)=-\eta \leqslant 0$ ensures that the system is UGAS with a guaranteed decay rate. Another example is provided in Section 4.

Let $z=(x, \hat{x}, \chi) \in \mathbb{R}^{n_{z}}$ with $n_{z}=2 n_{x}+n_{\chi}$. We model the closed-loop system (1)-(2) (and possibly auxiliary dynamics for $\chi$ ) as an impulsive system like in [9], which 
gives

$$
\begin{aligned}
\dot{z} & =g(z), \text { for } t \in\left(t_{k}, t_{k+1}\right] \\
z\left(t_{k}^{+}\right) & =b\left(z\left(t_{k}\right)\right) \\
t_{0} & =0 \\
t_{k+1} & =\inf \left\{t>t_{k} \mid \Gamma(z(t))>0\right\}
\end{aligned}
$$

for $k \in \mathbb{N}_{0}$, and appropriate $g: \mathbb{R}^{n_{z}} \rightarrow \mathbb{R}^{n_{z}}$ and $b$ : $\mathbb{R}^{n_{z}} \rightarrow \mathbb{R}^{n_{z}}$. In case $n_{\chi}=0$, we have that

$$
g(z)=\left[\begin{array}{c}
f(x, k(\hat{x})) \\
0
\end{array}\right] \text { and } b(z)=\left[\begin{array}{l}
x \\
x
\end{array}\right]
$$

For the definition of the functions $g$ and $b$ in case $n_{\chi} \neq 0$ we refer to Section 4 for an example.

Solutions to (3) are interpreted as follows. In between the event times $t_{k}, k \in \mathbb{N}$, determined by (3c), the system evolves according to the differential equation (3a), where $z\left(t_{k}^{+}\right)$given by the update $(3 \mathrm{~b})$ denotes the starting point for the solution to $(3 \mathrm{a})$ in the interval $\left(t_{k}, t_{k+1}\right], k \in$ $\mathbb{N}$. Hence, the solutions we consider are left-continuous signals. Note that $t_{0}=0$, and hence, we start with an update according to $(3 \mathrm{~b})$.

Remark 1 The analysis presented in this paper is based on system (3). Therefore, our design applies to any CETC configuration that can be written in the format of (3), including the case where the control input u in (1) is generated by a dynamic controller. The states of the controller would then be incorporated in the vector $x$ and we would obtain a model of the form (3). Similarly, the case in which the controller is not implemented using zero-order-hold functions can be considered as long as the problem can be modeled by (3). For instance, when using the model-based technique of [16], $\hat{x}$ would be equal to $x_{s}$ in [16], which is the model-based estimate of $x$.

In order to transform the CETC system (3) into a PETC system, we require the following three assumptions.

Assumption 1 There exists a nonempty set $\Omega \subseteq \mathbb{R}^{n_{z}}$ such that for all $z_{0} \in \Omega$, all corresponding solutions $z\left(t, z_{0}\right)$ to $(3)$ are defined for all time $t \in \mathbb{R}_{\geqslant 0}$ and satisfy $z\left(t, z_{0}\right) \in \Omega$ for all $t \in \mathbb{R}_{\geqslant 0}$.

Assumption 1 holds for most CETC designs proposed in literature, including $[1,5,7,11,13,15,16,22,24,25]$. It implies that the set $\Omega$ is forward invariant for the closedloop CETC system (3) (i.e., that all solutions starting in $\Omega$ remain in $\Omega$ for all $t \in \mathbb{R}_{\geqslant 0}$ ), and rules out finite escape times and Zeno-behaviour (an infinite number of events in a finite time interval) that would prevent global existence of all solutions starting in $\Omega$. Note that we do not exclude Zeno behavior or finite escape times for solutions starting outside $\Omega$.
Because the system starts with an update according to $(3 \mathrm{~b})\left(\right.$ as $\left.t_{0}=0\right)$, Assumption 1 also implies that the function $b$ satisfies $b(\Omega) \subseteq \Omega$. Hence, solutions do not leave the set $\Omega$, even if we allow the generation of events when $\Gamma(z(t))<0$, which will typically be the case in the PETC implementation that we envision.

Assumption 2 There exists $T>0$ such that the jumps induced by $\Gamma$ on the CETC system (3) are spaced by at least $T$ units of time, i.e., for all $z_{0} \in \Omega$ and all solutions $z\left(\cdot, b\left(z_{0}\right)\right)$ to $(3)$ it holds that

$$
\inf \left\{t>0 \mid \Gamma\left(z\left(t, b\left(z_{0}\right)\right)\right)>0\right\} \geqslant T .
$$

Assumption 2 implies that there exists a uniform positive minimum inter-event time $T$ for the CETC system in the set $\Omega$. Sufficient conditions for this assumption to hold are given in [22], and most available event-triggering schemes in the literature either provide a positive lower bound on $T$ (e.g., $[7,22,24])$, or enforce a positive lower bound by design (e.g., $[1,5,22,25])$.

Assumption 3 There exists $p \in \mathbb{N}$ such that $\Gamma$ is $p$ times continuously differentiable on $\Omega, g$ is $(p-1)$-times continuously differentiable on $\Omega$, and there exist real numbers $c, \varsigma_{j}, j \in\{0,1, \ldots, p-1\}$, satisfying

$$
\mathcal{L}_{g}^{p} \Gamma(z) \leqslant \sum_{j=0}^{p-1} \varsigma_{j} \mathcal{L}_{g}^{j} \Gamma(z)+c
$$

for all $z \in \Omega$, where $\mathcal{L}_{g}^{j} \Gamma$ is the $j$-th Lie derivative of $\Gamma$ along the flow dynamics $\dot{z}=g(z)$, with $\mathcal{L}_{g}^{0} \Gamma=\Gamma$, $\left(\mathcal{L}_{g} \Gamma\right)(z)=\frac{\partial \Gamma}{\partial z} g(z)$ and $\mathcal{L}_{g}^{j} \Gamma=\mathcal{L}_{g}\left(\mathcal{L}_{g}^{j-1} \Gamma\right)$ for $j \in \mathbb{N}$.

Assumption 3 is a condition on the evolution of $\Gamma$ along the solution to (3a), i.e., along the solutions to the system between two successive updates. It plays a crucial role in our design as it allows us to upper-bound the evolution of $\Gamma$ by the solution to a linear system, as explained later in Section 3.2. A similar assumption is made in the context of self-triggered control in [2]. In case $\Omega$ is compact, inequality (5) can always be satisfied when $g$ and $\Gamma$ are $(p-1)$-times and $p$-times continuously differentiable, respectively, as it suffices to take $c=\max _{z \in \Omega} \mathcal{L}_{g}^{p} \Gamma(z)$ and $\varsigma_{j}=0$ for $j \in\{0,1, \ldots, p-1\}$ to ensure (5). However, this particular choice may be conservative and tighter estimates of $\mathcal{L}_{g}^{p} \Gamma(z)$ can be obtained by using the other terms $\mathcal{L}_{g}^{j} \Gamma(z)$ in the right-hand side of (5). The parameter $p$ may be increased to further reduce the conservatism of the upper bounds on $\mathcal{L}_{q}^{p} \Gamma(z)$ in (5) at the price of a higher computational complexity.

In this paper, we describe a method for designing PETC strategies for nonlinear system (1), given that a CETC scheme (2) has already been designed. We do this by 
redesigning the event function $\Gamma$ to a new event function $\tilde{\Gamma}$, while we keep using the pre-designed feedback law. The envisioned closed-loop PETC system is described by

$$
\begin{aligned}
\dot{z}= & g(z), \text { for } t \in\left(\tilde{t}_{k}, \tilde{t}_{k+1}\right] \\
z\left(\tilde{t}_{k}^{+}\right)= & b\left(z\left(\tilde{t}_{k}\right)\right) \\
\tilde{t}_{0}= & 0 \\
\tilde{t}_{k+1}= & \min \left\{t>\tilde{t}_{k} \mid\right. \\
& \tilde{\Gamma}(z(t)) \geqslant 0, t=n h, n \in \mathbb{N}\},
\end{aligned}
$$

where $h>0$ is the sampling period at which the triggering condition $\tilde{\Gamma}$ is evaluated. Our aim is to provide tools for the design of $h$ and $\tilde{\Gamma}$ to guarantee that $\Gamma$ remains non-positive along all solutions to (6) starting in $\Omega$, such that the stability and performance guarantees of the original CETC system (3) are preserved. To do so, we will use the following design requirement on $h$ and $\tilde{\Gamma}$.

Design Requirement 1 For all $z_{0} \in \Omega$ such that $\tilde{\Gamma}\left(z_{0}\right)<0$ it holds that $\Gamma\left(z\left(t, z_{0}\right)\right)<0$ for all $t \in[0, h]$ for all solutions $z\left(\cdot, z_{0}\right)$ to $\dot{z}=g(z)$.

Remark 2 In general it is not possible to satisfy Design Requirement 1 by choosing $\tilde{\Gamma}=\Gamma$, see for example [21]. We will also demonstrate this in Section 4.

Remark 3 Based on Assumptions 1, 2, and 3, we present in the next section our method to transform the CETC system (3) into a PETC system (6). Compared to the conference version of this work [21], we provide the following improvements:

(1) We guarantee preservation of the control performance of the original CETC system (which we were not able to in [21]), see Theorem 8.

(2) We do not require that the set $\Omega$ is compact, see Assumption 1. As a result, we can now also apply our PETC design to CETC systems which have additional variables in the event-generator that are not forward invariant with regard to any compact set, such as timers or counters (see, e.g., [1, 5, 22, 25]).

(3) We do not require that all solutions $z\left(t, z\left(t_{j}^{s}\right)\right)$ to $\dot{z}=g(z)$ lie inside $\Omega$ for all $t \in[0, h]$. Consequently, we do not require [21, Assumption 3], which basically states that $\Omega$ is a forward invariant set for the system $\dot{z}=g(z)$ (without resets), which would mean that the plant already satisfies the desired control performance in open-loop.

These observations reveal a significant relaxation with respect to [21].

Note that items (1) and (2) also support our claims with respect to [23].

\section{Main results}

\subsection{Sampling period selection}

Under the PETC strategy, the input can be updated only when the triggering condition is evaluated, that is, every $h$ units of time. Hence, an event should be triggered at a sampling time $t=n h, n \in \mathbb{N}$, before $\Gamma$ becomes positive, and thus it is necessary that the sampling interval $h$ is less than the minimum inter-event time $T$ of the CETC system (which exists in view of Assumption 2).

In this way, after a jump, we know that $\Gamma$ will remain non-positive at least until the next sampling instant, and that Design Requirement 1 may be satisfied. Therefore, we select $h$ such that

$$
0<h \leqslant T
$$

with $T$ as in (4).

Remark 4 We do not need to verify the triggering condition for the next $\left\lfloor\frac{T}{h}\right\rfloor$ sampling instants following a control input update due to Assumption 2, which allows to reduce the computational load of the event-trigger.

As we aim at guaranteeing that $\Gamma$ remains non-positive along the solutions in $\Omega$ of the PETC system, we would like to verify at each sample time $t_{j}^{s}=j h, j \in \mathbb{N}_{0}$, whether the condition $\Gamma(z(t))>0$ may be satisfied for $t \in\left[t_{j}^{s}, t_{j+1}^{s}\right]$ (as then we will have to trigger an event at $\left.t_{j}^{s}\right)$. To do this, we would need to analyze the evolution of the triggering function $\Gamma$ along the solutions to $\dot{z}=g(z)$, which is difficult to do when $g(z)$ is a nonlinear function. We first propose to upper bound the evolution of $\Gamma$ on flows by a linear system.

\subsection{Analysis of the evolution of $\Gamma(z)$ on flows}

To upper bound the evolution of $\Gamma$ by a linear system, we resort to similar techniques as in [2], which rely on Assumption 3. This assumption allows to bound the evolution of $\Gamma$ by a linear differential equation as stated in the lemma below, which is a variation of [2, Lemma V.2]. The proof directly follows from the comparison lemma ([14, Lemma 3.4]).

Lemma 5 Consider any solution $z\left(\cdot, z_{0}\right)$ to $\dot{z}=g(z)$ with initial state $z_{0} \in \Omega$, and define $t^{*}:=\inf \left\{\tau \in \mathbb{R}_{\geqslant 0} \mid\right.$ $\left.z\left(\tau, z_{0}\right) \notin \Omega\right\}$. If Assumption 3 holds, then $\Gamma\left(z\left(t, z_{0}\right)\right) \leqslant$ $y_{1}\left(t, \mu^{p}\left(z_{0}\right)\right)$ for all $t \in\left[0, t^{*}\right]$, where $y_{1}\left(t, \mu^{p}\left(z_{0}\right)\right)$ is the first component of the solution to the linear differential equation

$$
\left\{\begin{array}{l}
\dot{y}_{j}=y_{j+1}, \quad j \in\{1,2, \ldots, p-1\} \\
\dot{y}_{p}=\sum_{j=0}^{p-1} \varsigma_{j} y_{j+1}+y_{p+1} \\
\dot{y}_{p+1}=0
\end{array}\right.
$$


with initial condition $y(0)=\mu^{p}\left(z_{0}\right)$ given by

$$
\mu^{p}\left(z_{0}\right)=\left(\Gamma\left(z_{0}\right), \mathcal{L}_{g} \Gamma\left(z_{0}\right), \ldots, \mathcal{L}_{g}^{p-1} \Gamma\left(z_{0}\right), c\right) .
$$

As formalized in the next theorem, for each initial state $z_{0} \in \Omega$, we can now check if $\Gamma$ remains non-positive for the complete sampling interval $[0, h]$, by checking the solution of the linear system (8) with initial condition (9).

Theorem 6 Suppose Assumptions 1 and 3 hold and that $z_{0} \in \Omega$, and consider the solution $y\left(\cdot, \mu^{p}\left(z_{0}\right)\right)$ to $(8)$ with initial condition (9). If $y_{1}\left(t, \mu^{p}\left(z_{0}\right)\right)<0$ for all $t \in[0, h]$, then for all solutions $z\left(\cdot, z_{0}\right)$ to $\dot{z}=g(z)$ with initial state $z_{0}$, it holds that $\Gamma\left(z\left(t, z_{0}\right)\right)<0$ for all $t \in[0, h]$.

PROOF. First, note that Assumption 1 is an invariance property of system (3), while here we consider the system $\dot{z}=g(z)$, which is system (3a) (without the discrete dynamics (3b) and (3c)). These are therefore two different dynamical systems, and the solutions to $\dot{z}=g(z)$, may leave $\Omega$, while those of (3) will not because of the well-designed discrete dynamics (jumps) in (3b) and $(3 \mathrm{c})$.

Consider any solution $z\left(\cdot, z_{0}\right)$ to $\dot{z}=g(z)$ with initial state $z_{0} \in \Omega$, and suppose that $y_{1}\left(t, \mu^{p}\left(z_{0}\right)\right)<0$ for all $t \in[0, h]$.

Case 1: $z\left(t, z_{0}\right) \in \Omega$ for all $t \in[0, h]$.

From Lemma 5 , it directly follows that $\Gamma\left(z\left(t, z_{0}\right)\right) \leqslant$ $y_{1}\left(t, \mu^{p}\left(z_{0}\right)\right)<0$ for all $t \in[0, h]$.

Case 2: $z\left(t, z_{0}\right) \notin \Omega$ for some $t \in[0, h]$.

Define $t^{*}:=\inf \left\{\tau \in[0, h] \mid z\left(\tau, z_{0}\right) \notin \Omega\right\}$ and $t_{1}:=$ $\left.\inf \left\{\tau>0 \mid \Gamma\left(z\left(\tau, z_{0}\right)\right)\right) \geqslant 0\right\}$. For all $t \in\left[0, t_{1}\right)$, it holds that $\Gamma\left(z\left(t, z_{0}\right)\right)<0$, and thus in this interval the solutions $z\left(t, z_{0}\right)$ to $\dot{z}=g(z)$ coincide with the solutions to the CETC system (3) with initial condition $z(0)=z_{0}$. As $\Omega$ is forward invariant for CETC system (3), it follows that $z\left(t, z_{0}\right) \in \Omega$ for all $t \in\left[0, t_{1}\right)$, which leads to the observation that $t_{1} \leqslant t^{*} \leqslant h$. Furthermore, $\Gamma\left(z\left(t_{1}, z_{0}\right)\right)=0$ as $\Gamma$ is continuous in view of Assumption 3. Finally, since according to Lemma 5, $y_{1}\left(t, \mu^{p}\left(z_{0}\right)\right) \geqslant \Gamma\left(z\left(t, z_{0}\right)\right)$ for all $t \in\left[0, t^{*}\right)$ we will have that $y_{1}\left(t_{1}, \mu^{p}\left(z_{0}\right)\right) \geqslant 0$, which is in contradiction with the assumptions of the theorem. As a result, Case 2 cannot occur.

Remark 7 The self-triggering formulas in [2] tend to provide accurate estimates of the time instants when $\Gamma$ becomes positive provided the bound (5) is tight, which may be difficult to achieve in practice. In the proposed PETC approach, the evolution of $\Gamma$ is investigated over shorter horizons than in STC, namely at time-intervals of length $h$. Hence, the bound in (5) does not necessarily need to be accurate to provide satisfactory results, as we expect these estimates to be tighter whenever times are shorter based on previous experience [2].

\subsection{Design of $\tilde{\Gamma}$}

The analytic expression of $y_{1}\left(t, \mu^{p}\left(z_{0}\right)\right)$ is given by

$$
y_{1}\left(t, \mu^{p}\left(z_{0}\right)\right)=C_{p} e^{A_{p} t} \mu^{p}\left(z_{0}\right)
$$

with

$$
A_{p}=\left[\begin{array}{ccccc}
0 & 1 & \cdots & 0 & 0 \\
\vdots & & \ddots & \vdots & \\
0 & 0 & \cdots & 1 & 0 \\
\varsigma_{0} & \varsigma_{1} & \cdots & \varsigma_{p-1} & 1 \\
0 & 0 & \cdots & 0 & 0
\end{array}\right] \text { and } C_{p}=\left[\begin{array}{c}
1 \\
0 \\
\vdots \\
0
\end{array}\right]^{\top}
$$

At each sampling instant $t_{j}^{s}, j \in \mathbb{N}_{0}$, the current state $z\left(t_{j}^{s}\right)$ is measured and a transmission should occur if $y_{1}\left(t, \mu^{p}\left(z\left(t_{j}^{s}\right)\right)\right) \geqslant 0$ for some $t \in[0, h]$, as then $\Gamma\left(z\left(t, z\left(t_{j}^{s}\right)\right)\right)$ may be positive for some $t \in[0, h]$. Otherwise, according to Theorem 6 a transmission is not necessary to satisfy Design Requirement 1 . However, verifying whether $y_{1}\left(t, \mu^{p}\left(z\left(t_{j}^{s}\right)\right)\right)<0$ for all $t \in[0, h]$, involves an infinite number of conditions and is computationally infeasible. In [21], this problem was (approximately) solved by evaluating $y_{1}\left(t, \mu^{p}\left(z\left(t_{j}^{s}\right)\right)\right)$ only at a finite number of points in the interval $[0, h]$. The price paid for this solution is that the non-positivity of $\Gamma(z(t))$ along the solutions of (6) can no longer be ensured, but only approximated.

The solution we propose here satisfies Design Requirement 1 , and thus guarantees non-positivity of $\Gamma(z(t))$ for all $t \in \mathbb{R}_{\geqslant 0}$, thereby preserving the stability and performance guarantees of the CETC system. Our method uses convex techniques to overapproximate the set $\left\{y_{1}\left(t, \mu^{p}\left(z\left(t_{j}^{s}\right)\right)\right) \mid t \in[0, h]\right\}$ in a computationally tractable manner.

To find a convex overapproximation of (10), we first define

$$
\Phi=\left\{C_{p} e^{A_{p} t} \mid t \in[0, h]\right\} .
$$

By using overapproximation techniques as described in [12], the set $\Phi$ of matrices can be embedded as

$$
\Phi \subseteq\left\{\sum_{i=1}^{N} \alpha_{i}\left(F_{i}+G_{i} \Delta H_{i}\right) \mid \alpha \in \mathcal{A}, \Delta \in \Delta\right\}
$$

in which $F_{i} \in \mathbb{R}^{1 \times p}, G_{i} \in \mathbb{R}^{1 \times q}, H_{i} \in \mathbb{R}^{q \times p}$ are suitably constructed matrices, $N$ is the number of vertices in the polytopic overapproximation (which can be freely selected), $\boldsymbol{\Delta}$ is a specific set of structured matrices in 
$\mathbb{R}^{q \times q}$ with norm bound $\|\Delta\| \leqslant 1, \alpha:=\left(\alpha_{1}, \ldots \alpha_{N}\right)$, and

$$
\mathcal{A}=\left\{\alpha \in \mathbb{R}_{\geqslant 0}^{N} \mid \sum_{i=1}^{N} \alpha_{i}=1\right\} .
$$

In [12], overapproximations as in (12) with $\Delta$ absent $\left(G_{i}=0\right.$ and $\left.H_{i}=0\right)$ are also given.

As $y_{1}\left(t, \mu^{p}\left(z_{0}\right)\right) \in \Phi \mu^{p}\left(z_{0}\right)$ for $t \in[0, h]$ and (12), it follows that $y_{1}\left(t, \mu^{p}\left(z_{0}\right)\right)<0$ for all $t \in[0, h]$ if

$$
\max \left\{\sum_{i=1}^{N} \alpha_{i}\left(F_{i}+G_{i} \Delta H_{i}\right) \mu^{p}\left(z_{0}\right) \mid \alpha \in \mathcal{A}, \Delta \in \boldsymbol{\Delta}\right\}<0,
$$

which holds when

$$
F_{i} \mu^{p}\left(z_{0}\right)+\left\|G_{i}\right\|\left\|H_{i} \mu^{p}\left(z_{0}\right)\right\|<0 \text { for all } i \in \bar{N},
$$

where we used that $\|\Delta\| \leqslant 1$. Consequently, we can choose $\tilde{\Gamma}$ in $(6 \mathrm{c})$ as

$$
\tilde{\Gamma}(z):=\max _{i \in N}\left\{F_{i} \mu^{p}(z)+\left\|G_{i}\right\|\left\|H_{i} \mu^{p}(z)\right\|\right\}
$$

to obtain the guarantee $\Gamma\left(z\left(t, z_{0}\right)\right) \leqslant 0$ for all $t \in \mathbb{R}_{>0}$, $z_{0} \in \Omega$, where $z$ is the corresponding solution to $(6)$, as formalized in the next theorem, whose proof directly follows from Theorem 6 and the developments above.

Theorem 8 Suppose Assumptions 1, 2, and 3 hold and consider the PETC system (6). If $h$ satisfies (7) and $\tilde{\Gamma}$ is defined by (15), then the set $\Omega$ is forward invariant for the system (6), and for any solution $z$ to (6) with initial state $z_{0} \in \Omega$ it holds that $\Gamma(z(t)) \leqslant 0$ for all $t \in \mathbb{R}_{>0}$.

PROOF. Define $z_{P}\left(t, z_{0}\right)$ as the solution to the PETC system (6), and $z_{C}\left(t, z_{0}\right)$ as the solution to the CETC system (3), both starting in $z_{0} \in \Omega$. Hence, $z_{P}\left(t_{0}, z_{0}\right)=$ $z_{C}\left(t_{0}, z_{0}\right)=z_{0}$. At $t=0$, both (3) and (6) start with an update. Hence, $z_{P}\left(t_{0}^{+}, z_{0}\right)=z_{C}\left(t_{0}^{+}, z_{0}\right)=b\left(z_{0}\right)$. Next, due to Assumption 2 and $h$ satisfying (7), it follows that $z_{P}\left(t, z_{0}\right)=z_{C}\left(t, z_{0}\right)$ for all $t \in[0, h]$. At $t=h$, the PETC system (6) may or may not generate an event. In case an event is generated by the PETC system (6), we have that $\tilde{t}_{1}=h<T$, and hence that $\tilde{t}_{1}<t_{1}$. In case an event is not generated by the PETC system (6) at $t=h$, it still follows that $\tilde{t}_{1} \leqslant t_{1}$, as $\tilde{\Gamma}$ defined by (15) satisfies Design Requirement 1 . The above proves that $z_{P}\left(t, z_{0}\right)=z_{C}\left(t, z_{0}\right)$ for all $t \in\left[0, \tilde{t}_{1}\right]$, and hence that $\Gamma\left(z_{P}\left(t, z_{0}\right)\right) \leqslant 0$ for all $t \in\left(0, \tilde{t}_{1}\right]$ as the solution to (3) satisfies $\Gamma\left(z_{C}\left(t, z_{0}\right)\right) \leqslant 0$ for all $t \in \mathbb{R}_{>0}$.

As $b(\Omega) \subseteq \Omega$, the same reasoning can be used to show that $\Gamma\left(z_{P}\left(t, z_{0}\right)\right) \leqslant 0$ for all $t \in\left(\tilde{t}_{1}, \tilde{t}_{2}\right]$, and by induction, that $\Gamma\left(z_{P}\left(t, z_{0}\right)\right) \leqslant 0$ for each interval $\left(\tilde{t}_{k}, \tilde{t}_{k+1}\right], k \in \mathbb{N}$.
The proof is completed by observing that $\lim _{k \rightarrow \infty} t_{k}=$ $\infty$.

Note that the redesigned event function $\tilde{\Gamma}$ may become positive in between sampling instants along solutions to (6). However, when $\tilde{\Gamma}\left(z\left(t_{j}^{s}\right)\right)<0$ at sample time $t_{j}^{s}, j \in$ $\mathbb{N}_{0}$, our design of $\tilde{\Gamma}$ given by (15) ensures that $\Gamma(z(t))<0$ will be satisfied for all $t \in\left[t_{j}^{s}, t_{j+1}^{s}\right]=\left[t_{j}^{s}, t_{j}^{s}+h\right]$. Alternatively, when $\tilde{\Gamma}\left(z\left(t_{j}^{s}\right)\right) \geqslant 0$, then our periodic eventgenerator (6c) triggers an event, after which Assumption 2 ensures that $\Gamma(z(t)) \leqslant 0$ will be satisfied for all $t \in\left[t_{j}^{s}, t_{j}^{s}+T\right]$. Hence, by selecting $h<T$, our proposed periodic event-generator ensures that $\Gamma(z(t)) \leqslant 0$ for all $t \in \mathbb{R}_{>0}$, and thus preserves the control performance guarantees of the original CETC system (3).

Remark 9 When the numerical complexity of calculating $\mu^{p}(z)$ is $\mathcal{O}(\phi(p))$, for some function $\phi$, then evaluating the event function (15) has numerical complexity $\mathcal{O}(N q p+\phi(p))($ or $\mathcal{O}(N p+\phi(p))$ when $\Delta$ is absent $)$. Thus, from a computational point of view, it makes sense to choose $p$ and $N$ small. On the other hand, choosing $p$ and $N$ large usually increases the accuracy of the overapproximation of the evolution of $\Gamma$ along the solutions to $\dot{z}=g(z)$ in view of [2] and [12], which leads to larger inter-event times. As such, there is a trade-off between computation and communication, as is also apparent from the example in Section 4 below.

Remark 10 The plant (1) can be extended to include process disturbances $w$ (i.e., $\dot{x}(t)=f(x(t), u(t), w(t))$ ) as long as the continuous event-triggering law (2c) also satisfies Assumptions 1 and 2. In order to satisfy Assumption 3 we then either require that $w$ does not show up in $\mathcal{L}_{g}^{j} \Gamma(z)$ in (5) for $j \in\{1,2, \ldots, p\}$ (which might be the case when $\Gamma(z)$ does not depend on the complete plant state $x$ ), or we need to know (estimates of) bounds on $w$ and of its time-derivatives which show up in (5).

\section{Illustrative example}

Consider the following example inspired by [19], in which the plant is given by

$$
\dot{x}(t)=x^{2}(t)-x^{3}(t)+u(t),
$$

and the controller by

$$
u(t)=-2 \hat{x}(t),
$$

where $\hat{x}$ is the sampled version of $x$ as in (2a). We design a continuous event-triggering condition as in [1]. The idea is to wait a fixed amount of time $T>0$ before checking a state-dependent criterion of the same form as in [24]. We introduce an extra variable $\chi \in \mathbb{R}_{\geqslant 0}$ to keep track of the 
time between two events. Hence, $\chi$ evolves according to $\dot{\chi}(t)=1$ for $t \in\left[t_{k}, t_{k+1}\right)$ and $\chi\left(t_{k}^{+}\right)=0$. The function $\Gamma$ in (2) is given in this case by

$$
\Gamma(x, \hat{x}, \chi)=\min \left\{\chi-T, \gamma^{2}|\hat{x}-x|^{2}-\delta(x)\right\}
$$

with parameter $\gamma \in \mathbb{R}_{>0}$ and $\delta$ a positive definite function to be designed. Hence, the CETC system (3) is given by $z=[x, \hat{x}, \chi]^{\top}, g(z)=\left[x^{2}-x^{3}-2 \hat{x}, 0,1\right]^{\top}$, $b(z)=[x, x, 0]^{\top}$, and $\Gamma$ as in (18). Note that this CETC system satisfies Assumption 2 by design for any set $\Omega$.

To construct $\tilde{\Gamma}$, we have verified that the conditions required in [1] hold, by following similar lines as in [19, Example 1]. We have taken the Lyapunov function $R(z)=V(x)+\max \left\{0, \lambda \zeta(\chi) W^{2}(\hat{x}-x)\right\}$, with $V(x)=\sigma^{2}\left(\frac{\alpha}{2} x^{2}+\frac{\beta}{4} x^{4}\right), W(\hat{x}-x)=|\hat{x}-x|$, $\zeta: \mathbb{R}_{\geqslant 0} \rightarrow \mathbb{R}$ the solution to

$$
\dot{\zeta}=-2 L \zeta-\lambda\left(\zeta^{2}+1\right), \quad \zeta(0)=\theta^{-1},
$$

and parameters $\sigma=1, \alpha=\beta=3, L=2, \bar{\rho}=0.1$, $\eta=2, \lambda=\sigma \sqrt{\alpha^{2}+\beta^{2}+\sigma^{-2} \bar{\rho}+\eta}$, and $\theta=0.9$, and we have obtained that the choice $T=0.010, \delta(x)=\bar{\delta} x^{2}$ with $\bar{\delta}=0.5$, and $\gamma=\sigma \sqrt{\alpha^{2}+\beta^{2}+\sigma^{-2} \bar{\rho}}$, guarantees uniform global asymptotic stability of the set $\mathcal{A}:=\{z \in$ $\left.\mathbb{R}^{n_{z}} \mid x=0\right\}$ for the CETC system.

The evolution of $\Gamma$ along the solution to the CETC system with initial condition $z(0)=z_{0}=[0.3,0.3,0]^{\top}$ is shown in Figure 4. This figure also includes a simulation

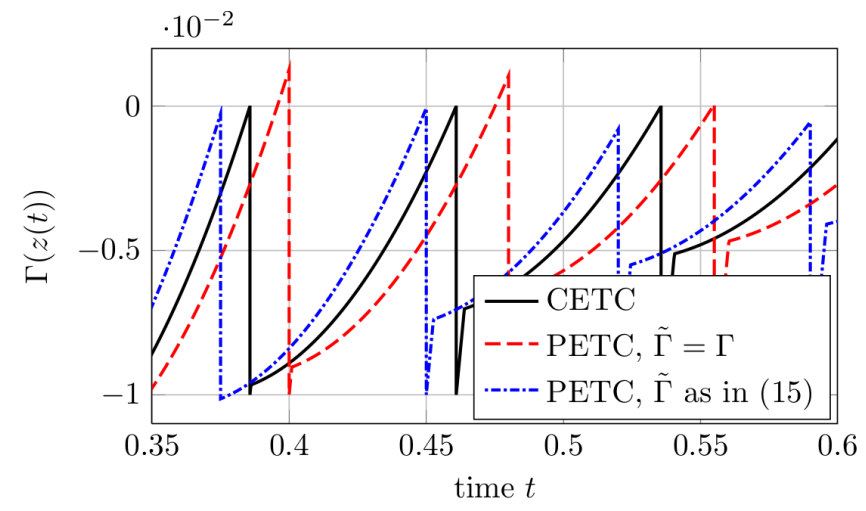

Fig. 1. Evolution of $\Gamma(z(t))$ along the solution to the CETC system, the PETC implementation with $\tilde{\Gamma}=\Gamma$, and the PETC implementation with $\tilde{\Gamma}$ as in (15).

of the emulated PETC implementation with $h=0.005$ and $\tilde{\Gamma}=\Gamma$. Clearly, this PETC implementation does not ensure that $\Gamma(z(t)) \leqslant 0$ for all $t \in \mathbb{R}_{>0}$, and thus does not preserve the control performance guarantees of the CETC system.
To overcome this issue, we construct $\tilde{\Gamma}$ as in (15). For this purpose we need $\Gamma$ to be $p$-times continuously differentiable, for some $p \in \mathbb{N}$, which is currently not the case in view of (18). Still, we can apply the procedure of Section 3.2 for $\Gamma_{1}=\chi-T$ and $\Gamma_{2}=\gamma^{2}|\hat{x}-x|^{2}-\delta(x)$ separately. The case of $\Gamma_{1}$ is trivial as we know that it is violated after $T$ units of time. We proceed by constructing $\tilde{\Gamma}_{2}$ for $\Gamma_{2}$. We have verified numerically using SOSTOOLS [20] that for $\Gamma_{2}$, Assumption 3 holds in the forward invariant set

$$
\Omega:=\{z \mid R(z) \leqslant 0.15\} .
$$

The obtained values of $c$ and $\varsigma_{i}, i \in\{0,1, \ldots, p-1\}$, for different values of $p$ are reported in Table 1.

Table 1

Parameters of Assumption 3 for $\Gamma_{2}$ in the invariant set (20).

\begin{tabular}{c|ccccc} 
& $c$ & $\varsigma_{0}$ & $\varsigma_{1}$ & $\varsigma_{2}$ & $\varsigma_{3}$ \\
\hline$p=2$ & 359.7 & -26.46 & 3.085 & & \\
$p=3$ & 0 & 222.8 & -36.37 & 10.1 & \\
$p=4$ & 0 & 4384 & -645.9 & 145.3 & -17.36
\end{tabular}

Finally, we design the PETC strategy by following the procedure in Section 3 and by using the gridding and norm bounding (GNB) overapproximation technique as described in [12, Section III], with $N$ equally distanced grid points.

A simulation of this redesigned PETC implementation with $p=3$ and $N=2$ is shown in Figure 4 (again with $z(0)=z_{0}$ and $\left.h=0.005\right)$. Clearly, the redesigned PETC implementation does ensure that $\Gamma(z(t)) \leqslant 0$ for all $t \in \mathbb{R}_{>0}$, and the control performance of the CETC system is preserved.

In Table 2, the average inter-event time $\tau_{\text {avg }}$ for the first 10 events of the proposed PETC implementation is shown for different parameter settings. The average inter-event time for the first 10 events of the original CETC system is $\tau_{a v g}=0.0757$. As expected, choosing

Table 2

Average inter-event time $\tau_{\text {avg }}$ for the first 10 events of the PETC implementation with $\tilde{\Gamma}$ as in (15).

\begin{tabular}{c|cccc} 
& $h=T$ & $h=T / 2$ & $h=T / 4$ & $h=T / 8$ \\
\hline$p=2, N=2$ & 0.0100 & 0.0380 & 0.0627 & 0.0715 \\
$p=2, N=3$ & 0.0200 & 0.0495 & 0.0672 & 0.0731 \\
$p=2, N=5$ & 0.0240 & 0.0535 & 0.0685 & 0.0735 \\
$p=2, N=9$ & 0.0260 & 0.0540 & 0.0690 & 0.0735 \\
\hline$p=3, N=2$ & 0.0700 & 0.0730 & 0.0745 & 0.0751 \\
$p=3, N=9$ & 0.0700 & 0.0730 & 0.0745 & 0.0751 \\
\hline$p=4, N=9$ & 0.0700 & 0.0730 & 0.0745 & 0.0751
\end{tabular}

$h$ smaller leads to a better approximation of the CETC 
system, and thus the inter-event times of the PETC implementation approach those of the CETC implementation. Similarly, increasing $N$ leads to larger inter-event times when $p=2$, and increasing $p$ to $p=3$ also leads to a large improvement (especially for large values of $h$ ). However, increasing $p$ or $N$ beyond $p=3, N=2$, does not further improve the results.

\section{Conclusions}

We have presented a systematic method for designing periodic event-triggered controllers for nonlinear systems, given that a continuous event-triggered controller is already available. Using convex overapproximation techniques, we were able to preserve the control performance guarantees of the given CETC system.

\section{References}

[1] M. Abdelrahim, R. Postoyan, J. Daafouz, and D. Nešić. Stabilization of nonlinear systems using event-triggered output feedback controllers. IEEE Trans. Autom. Control, 2016.

[2] A. Anta and P. Tabuada. Exploiting isochrony in self-triggered control. IEEE Trans. Autom. Control, 57(4):950-962, 2012.

[3] C.G. Cassandras. The event-driven paradigm for control, communication and optimization. J. Control and Decision, 1(1):3-17, 2014.

[4] R. Cogill. Event-based control using quadratic approximate value functions. In 48th IEEE Conf. Decision and Control and Chinese Control Conf., pages 5883-5888, 2009.

[5] V.S. Dolk, D.P. Borgers, and W.P.M.H. Heemels. Outputbased and decentralized dynamic event-triggered control with guaranteed $\mathcal{L}_{p}$-gain performance and Zeno-freeness. IEEE Trans. Autom. Control, 62(1):34-49, 2017.

[6] A. Eqtami, D.V. Dimarogonas, and K.J. Kyriakopoulos. Event-triggered control for discrete-time systems. In Proc. Amer. Control Conf., pages 4719-4724, 2010.

[7] A. Girard. Dynamic triggering mechanisms for eventtriggered control. IEEE Trans. Autom. Control, 60(7):1992-1997, 2015.

[8] W.P.M.H. Heemels and M.C.F. Donkers. Modelbased periodic event-triggered control for linear systems. Automatica, 49(3):698-711, 2013.

[9] W.P.M.H. Heemels, M.C.F. Donkers, and A.R. Teel. Periodic event-triggered control for linear systems. IEEE Trans. Autom. Control, 58(4):847-861, 2013.

[10] W.P.M.H. Heemels, G.E. Dullerud, and A.R. Teel. $\mathcal{L}_{2}$-gain analysis for a class of hybrid systems with applications to reset and event-triggered control: A lifting approach. IEEE Trans. Autom. Control, 61(10):2766-2781, 2016.

[11] W.P.M.H. Heemels, J.H. Sandee, and P.P.J. Van Den Bosch. Analysis of event-driven controllers for linear systems. Int. J. Control, 81(4):571-590, 2008.

[12] W.P.M.H. Heemels, N. van de Wouw, R.H. Gielen, M.C.F. Donkers, L. Hetel, S. Olaru, M. Lazar, J. Daafouz, and S.I. Niculescu. Comparison of overapproximation methods for stability analysis of networked control systems. In Hybrid Systems: Computation and Control, pages 181-191, 2010.
[13] T. Henningsson, E. Johannesson, and A. Cervin. Sporadic event-based control of first-order linear stochastic systems. Automatica, 44(11):2890-2895, 2008.

[14] H.K. Khalil. Nonlinear Systems. Prentice-Hall, Upper Saddle River, NJ, USA, 3rd edition, 2002.

[15] L. Li and M. Lemmon. Weakly coupled event triggered output feedback system in wireless networked control systems. In Allerton Conf. Commun., Control and Computing, 2011.

[16] J. Lunze and D. Lehmann. A state-feedback approach to event-based control. Automatica, 46(1):211-215, 2010.

[17] M. Miskowicz. Send-on-delta concept: An event-based data reporting strategy. Sensors, 6(1):49-63, 2006.

[18] A. Molin and S. Hirche. On the optimality of certainty equivalence for event-triggered control systems. IEEE Trans. Autom. Control, 58(2):470-474, 2013.

[19] D. Nešić, A.R. Teel, and D. Carnevale. Explicit computation of the sampling period in emulation of controllers for nonlinear sampled-data systems. IEEE Trans. Autom. Control, 54(3):619-624, 2009.

[20] A. Papachristodoulou, J. Anderson, G. Valmorbida, S. Prajna, P. Seiler, and P.A. Parrilo. SOSTOOLS: Sum of squares optimization toolbox for MATLAB. http://arxiv.org/abs/1310.4716, 2013. Available from http://www.cds.caltech.edu/sostools.

[21] R. Postoyan, A. Anta, W.P.M.H. Heemels, P. Tabuada, and D. Nešić. Periodic event-triggered control for nonlinear systems. In 52nd IEEE Conf. Decision and Control, pages 7397-7402, 2013.

[22] R. Postoyan, P. Tabuada, D. Nešić, and A. Anta. A framework for the event-triggered stabilization of nonlinear systems. IEEE Trans. Autom. Control, 60(4):982-996, 2015.

[23] R.G. Sanfelice and A.R. Teel. Lyapunov analysis of sampleand-hold hybrid feedbacks. In 45th IEEE Conf. Decision and Control, pages 4879-4884, 2006.

[24] P. Tabuada. Event-triggered real-time scheduling of stabilizing control tasks. IEEE Trans. Autom. Control, 52(9):1680-1685, 2007.

[25] P. Tallapragada and N. Chopra. Decentralized eventtriggering for control of nonlinear systems. IEEE Trans. Autom. Control, 59(12):3312-3324, 2014.

[26] W. Wang, R. Postoyan, D. Nešić, and W. P. M. H. Heemels. Stabilization of nonlinear systems using statefeedback periodic event-triggered controllers. In 55th IEEE Conf. Decision and Control, pages 6808-6813, 2016.

[27] J.K. Yook, D.M. Tilbury, and N.R. Soparkar. Trading computation for bandwidth: reducing communication in distributed control systems using state estimators. IEEE Trans. Control Syst. Technol., 10(4):503-518, 2002. 